Asian J. Med. Biol. Res. 2018, 4 (1), 95-104; doi: 10.3329/ajmbr.v4i1.36827

\author{
Asian Journal of \\ Medical and Biological Research \\ ISSN 2411-4472 (Print) 2412-5571 (Online) \\ www.ebupress.com/journal/ajmbr
}

\title{
Article \\ Morphometric characterization of Black Bengal Goat under farming condition in Bangladesh
}

\author{
M.A. Jalil ${ }^{1}$, M.P. Choudhury ${ }^{1}$, M.M. Kabir ${ }^{2}$ and M.A. Habib ${ }^{1}$ \\ ${ }^{1}$ Bangladesh Livestock Research Institute, Savar, Dhaka-1341, Bangladesh \\ ${ }^{2}$ Jahangirnagar University, Savar, Dhaka-1342, Bangladesh \\ *Corresponding author: Dr. Md. Abdul Jalil, Bangladesh Livestock Research Institute, Savar, Dhaka-1341, \\ Bangladesh. Phone: +8801711155062; E-mail: jalilgen@gmail.com
}

Received: 07 March 2018/Accepted: 21 March 2018/ Published: 29 March 2018

\begin{abstract}
The study was undertaken to characterize of Black Bengal Goat (BBG) under farming condition. Data on the different parameters were collected during July 2006 to June 2013 at BLRI goat research farm. A total of 299 animals of different ages from birth to 36 months of both sexes were included. All type of measurements were taken when goats standing freely. All measurements were taken in metric unit. Data were analyzed by SPSS version 17.0 statistical computer program. In BLRI goat herd, four different types of coat color were observed in BBG i.e. Black, Black and white, Black and brown and completely white. Body length is higher in males than that of females for all generation and age group. Male goats had higher heart girth than that of female goats irrespective of age and generations. Average adult ( $>24$ months age) body weight of male and female goats as $29.9 \pm 1.76$ and $23.6 \pm 0.81 \mathrm{~kg}$, respectively. Wither height was higher in adult males than females for same age. Left and right horn length in both sexes ranged from 3.6 to $13.2 \mathrm{~cm}$. Female goats had higher ear length than male goats. Tail length of Black Bengal goats ranged from $8.0 \pm 0.52$ to $11.1 \pm 0.43 \mathrm{~cm}$ and tail breadth ranged from $2.1 \pm 0.10$ to $3.6 \pm 0.43 \mathrm{~cm}$ for different age and sex group. Average male foreleg length in $>24$ months of age possessed higher than that of female. Average udder length and breadth of BBGs were $7.5 \pm 0.24,11.7 \pm 0.44$ and $14.3 \pm 0.46$ and $5.9 \pm 0.16,7.4 \pm 0.28$ and $7.8 \pm 0.23 \mathrm{~cm}$, respectively for age groups $6-12$, $12-24$ and $>24$ months of age. The average testis length and breadth in adult males were $9.1 \pm 0.18,7.5 \pm 0.48$ and $9.8 \pm 0.49$ and $6.8 \pm 0.13,5.2 \pm 0.27$ and $6.3 \pm 0.32$, cm respectively for $6-12,12-24$ and $>24$ months of ages groups.
\end{abstract}

Keywords: Black Bengal Goat; morphometric; characterization

\section{Introduction}

Among the Asiatic countries, Bangladesh has the fourth highest population of goats and there are about 14.8 million goats in the country. Bangladesh has only one goat breed of its own, popularly known as the Black Bengal Goat (BBG). It is estimated that more than $90 \%$ of goat population in Bangladesh comprised the Black Bengal, the remainder being Jamunapari and their crosses (Husain, 1993). It is also observed that there are wide variations in color, body size and weights of goats found in different locations. The BBG is mostly black in color, which comprises $69 \%$ of the total goat population. However, white stripe on black (13\%), brown (5\%), solid white (4\%), black with white patches or brown with white or brown with black (9\%) also found (Chowdhury, 2002). As a reservoir of genetic resource, BBG occupies very illustrious position among the dwarf meat type goat of the world. Higher prolificacy, fertility, resistance against common diseases, adaptability to stressful adverse environmental condition, early maturity, seasonality and superiority in the kidding intensity are some of the outstanding features of BBG. Besides, it produces excellent quality flavored, tender and delicious meat and skin of extra ordinary quality for which there is tremendous demand all over the world (Devendra and Burns, 1983; Islam et al., 1991; Singh et al., 1991 and Husain, 1993). 
Prasad et al., 1981 reported that there was a correlation of body weight with length, height and heart girth in BBG. Correlation of body weight with heart girth was highest and this was followed by length and height, respectively. High relationship of body weight with chest girth was also observed by Singh et al., 1986 in BBG and by Mukherjee et al., 1979 in Grey and Brown Bengal goats. Husain et al., 1996 observed the existence of large variation within breed in terms of birth weight, mature body weight, growth rate and milk yield of dam which may be exploited for their immediate improvement through selective breeding. Relationship between body weight and length might be due to close association between weight and growth of muscles and bones leading to increase in length. Increase in chest girth includes the growth of bones, muscle and viscera.

But owing to small body size, meat produced by this breed does not fetch a high economic return. As BBGs are being reared primarily for meat production, body weight and growth rate could be considered as the most important factors. In spite of promises of BBG amongst the world goat genetic resource no substantial amount of work has yet been done on the morphometric evaluation and characterization. Considering the above facts it is the best tone to describe the BBG as a breed.

Therefore, the main objective of this study is to characterize the BBG on the basis of morphometric information about the breed for its better description.

\section{Materials and Methods}

\subsection{Source of data}

Data on the different parameters were collected during July 2006 to June 2013 from the study "Characterization of Black Bengal goat" at BLRI goat research farm. A total of 299 animals of different ages from birth to 36 months of both sexes were included which were collected from Bogra, Faridpur, Kishorgonj, Noakhali, Mymensingh, Jamalpur, Tangail, Manikgonj, Dhaka and Gazipur district. All type of measurements were taken when goats standing freely. All measurements were taken in metric unit.

Coat Color: Coat Color was observed on basis of hair color.

Body length: Body length was measured between points of shoulder and point of hip.

Heart girth: Circumference of the body immediately behind the shoulder.

Body weight: Body weight was taken with help of top loading balance before feeding of animals.

Wither height: Distance from the ground to the point of wither.

Head length and breadth: Head length is measured from the upper lip to the pole of head. Breadth is distance between two eyes.

Horn length and circumference: Horn length was measured between base of the horn and pin point of horn. Horn circumference was taken from middle point of horn.

Ear length and breadth: Ear length is the distance between the base and the pinna of ear. Ear breadth was measured at broadest portion of the ear.

Neck length and breadth: Neck length is the distance between the base of head and point of shoulder. Neck breadth was measured from the middle position of neck.

Tail length and breadth: Length was measured from the base of tail to the pinpoint of the tail without hair. Breadth was measured from middle portion of tail.

Leg length: Fore leg length is the distance from hoof to joint of shoulder and hind leg length is the distance from the hoof to elbow joint.

Udder length and breadth: Length was measured from base of teat to the attachment point of udder and belly. Breadth was measured from the middle portion of udder.

Teat length and circumference: Teat length was measured from the base of the teat to pin-point of teat. Circumference was measured from middle portion of teat.

Testis length and breadth: Distance between two-pin point of testes and breadth was measured from the middle portion of two testes.

\section{Results and Discussion}

\subsection{Coat color}

In BLRI goat herd, four different types of coat color were observed in BBG i.e. Black, Black and white, Black and brown and completely white. Maximum goats were black in color and the proportion chronologically reduced as Black and white, Black and brown and white accordingly (Table 1). Husain, 1993 reported that more than 90 percent goats of this country belong to Black Bengal with same incenses fancy in coat color. Majority of the remaining 10 percent of the species were exotic and its crosses with BBG. Husain et al., 1998 reported that Black Bengal goat is originally black in color that comprises $80 \%$ of the total goat population and the rest are white, brown, grey and mixed or spotted body color. Chowdhury, 2002 also reported that $69 \%$ Bengal goats are 
black, $13 \%$ white stripe on black, $5 \%$ brown, $4 \%$ solid white and $9 \%$ black with white patches or brown with white or brown with black.

\subsection{Body length}

Body length observed in this study according to generation, age group and sex had been presented in Table 2 . Body length measurement of BBG is shown in Figure 1. From the Table 2, it appears that body length is higher in males than that of females for all generation and age group. These findings are in agreement with the result of (Chowdhury and Faruque, 2001a). They showed that body length is higher in males than that of females. The range of body length of adult (12-24 months) BBG goat is 47 to $54 \mathrm{~cm}$, which is similar to the Husain, 1993 . These results were lower than of (Kibria and Kamal, 1990) who reported that body length of BBG ranged from 52 to $57 \mathrm{~cm}$. Singh et al., (1987) reported that the body length of both male and female Black Bengal goats at 12 months of age is 40.16 and $40.04 \mathrm{~cm}$ respectively, which is lower than the result of the present study in both the sexes. This result agrees with the result reported by (Bhattacharya et al., 1984), which was $44 \pm 0.72 \mathrm{~cm}$ in BBG.

\subsection{Heart girth}

Heart girth of male and female BBG at different age group and generation are presented in Table 2. Heart girth measurement of BBG is shown in Figure 1. From the Table 2, it appears that male goats had higher heart girth than that of female goats irrespective of age and generations. Heart girth of male and female goats in age groups 6-12, 12-24 and $>24$ months in $\mathrm{G}_{0}, \mathrm{G}_{1}, \mathrm{G}_{2}$ and $\mathrm{G}_{3}$ were $56.4 \pm 0.65$ and $51.7 \pm 0.48,61.6 \pm 1.02$ and $60.5 \pm 0.48$ and $72.3 \pm 1.35$ and $68.1 \pm 0.82 \mathrm{~cm}$, respectively. These results are in agreement with the result of (Chowdhury and Faruque, 2001b) who shown that heart girth of male and female BBG at $>12$ months of age is $73.2 \pm 1.33$ and $71.0 \pm 1.13 \mathrm{~cm}$, respectively.

\subsection{Body weight}

Body weights in male and female goats at different age groups and generations are presented in Table 3. In which shows average adult ( $>24$ months age) body weight of male and female goats as $29.9 \pm 1.76$ and $23.6 \pm 0.81$ $\mathrm{kg}$, respectively. Chowdhury and Faruque, 2001b reported that adult body weight of male and female goats are $33.44 \pm 1.43$ and $26.33 \pm 1.13 \mathrm{~kg}$, respectively but contradict with the result of Husain, 1993 who reported average body weight of BBG as $13.20 \mathrm{~kg}$. Devendra and Burns, 1983 stated that BBG has been classified as dwarf breed having body weight of 9 to $22 \mathrm{~kg}$ with a wither height of 30 to $95 \mathrm{~cm}$ for male and 40 to $90 \mathrm{~cm}$ for female. Higher body weight in the present herd of BBG is probably due to the selection and better environmental conditions particularly due to the better nutrition (Chowdhury and Farque, 2001a).

\subsection{Wither height}

Wither height of male and female goat at different age groups and generation are presented in Table 2. Wither height measurement of BBG is shown in Figure 1. From the Table 2, wither height was higher in adult males than females for same age. Wither height of male and female goats at 12 to 24 months of age in $G_{1}$ was $56.5 \pm 1.24$ and $51.9 \pm 0.82 \mathrm{~cm}$ and in $\mathrm{G}_{2}$ was $53.2 \pm 1.54$ and $49.6 \pm 0.85 \mathrm{~cm}$, respectively. Husain, 1993 reported that height at wither of adult BBG is $49.5 \mathrm{~cm}$, which was lower to the present findings. Chowdhury and Faruque, $2001 \mathrm{~b}$ also reported that wither height of adult ( $>12$ months) male goats is higher $(58.6 \pm 0.88 \mathrm{~cm})$ than female goats $(54.4 \pm 0.70 \mathrm{~cm})$. Wither height in both male and female BBG at 12 months of age to be 49.69 and $48.58 \mathrm{~cm}$, respectively reported by (Singh et al., 1987).

\subsection{Head length and breadth}

Head length and breadth of Black Bengal goats at different age group, sex and generation are presented in Table 3. Head length, breadth, Horn length, circumference and Horn distance measurement of BBG are shown in Figure 2. Head length and breadth was higher in male than female goat for all age groups and generation. In case of head length (Chowdhury and Faruque, 2001b) obtained the same results (cm). No such available report was found in literature on head length and breadth.

\subsection{Horn length and circumference}

Horn length and circumference of Black Bengal goat are shown in Table 4. Table 4 shows that left and right horn length in both sexes ranged from 3.6 to $13.2 \mathrm{~cm}$. The present findings are in agreement with the results of (Husain et al., 1998) who stated that both sexes have horn 6 to12 cm. Chowdhury and Faruque, 2001b also shown that adult male and female goats have horn 4 to $12 \mathrm{~cm}$. Horn circumference of both sexes of adult goat ranges from 3.9 to $9.7 \mathrm{~cm}$. 
Asian J. Med. Biol. Res. 2018, 4 (1)

Table 1. Coat color of Black Bengal goat according to age and generation.

\begin{tabular}{|c|c|c|c|c|c|c|}
\hline \multirow{2}{*}{$\begin{array}{l}\text { Color } \\
\text { phenotype }\end{array}$} & \multirow[t]{2}{*}{ Age } & \multicolumn{4}{|c|}{ Generations } & \multirow[t]{2}{*}{ Overall mean } \\
\hline & & $\mathbf{G}_{\mathbf{0}}$ & $\mathbf{G}_{1}$ & $\mathbf{G}_{2}$ & $\mathbf{G}_{3}$ & \\
\hline \multirow[t]{3}{*}{ Black (\%) } & $6-12 \mathrm{~m}$ & $34.78(24)$ & $79.41(25)$ & $65.63(32)$ & $50.00(43)$ & 57.46 \\
\hline & $12-24 \mathrm{~m}$ & 70.97 (22) & 77.5 (18) & $87.62(23)$ & $50.00(25)$ & 71.52 \\
\hline & $>24 \mathrm{~m}$ & $63.64(22)$ & $82.93(33)$ & - & - & 73.29 \\
\hline \multirow{3}{*}{$\begin{array}{l}\text { Black \& white } \\
(\%)\end{array}$} & $6-12 \mathrm{~m}$ & $52.17(26)$ & $20.59(23)$ & $25.00(24)$ & $50.00(36)$ & 36.94 \\
\hline & $12-24 \mathrm{~m}$ & $29.03(24)$ & $22.5(31)$ & $3.85(27)$ & $50.00(33)$ & 26.35 \\
\hline & $>24 \mathrm{~m}$ & $30.91(23)$ & $9.76(44)$ & $100(30)$ & - & 46.89 \\
\hline \multirow{3}{*}{$\begin{array}{l}\text { Black \& } \\
\text { brown (\%) }\end{array}$} & $6-12 \mathrm{~m}$ & $13.04(18)$ & - & $9.38(15)$ & - & 11.21 \\
\hline & $12-24 m$ & - & - & $3.85(14)$ & - & 3.85 \\
\hline & $>24 \mathrm{~m}$ & $5.45(17)$ & $7.32(16)$ & - & - & 6.39 \\
\hline \multirow[t]{3}{*}{ White (\%) } & $6-12 \mathrm{~m}$ & - & - & - & - & - \\
\hline & $12-24 m$ & - & - & 7.69 (12) & - & 7.69 \\
\hline & $>24 \mathrm{~m}$ & - & - & - & - & - \\
\hline
\end{tabular}

$\mathrm{G}_{0}, \mathrm{G}_{1}, \mathrm{G}_{2}$ and $\mathrm{G}_{3}$ means foundation stock, $1^{\text {st }}$ generation, $2^{\text {nd }}$ generation and $3^{\text {rd }}$ generation, respectively; - means samples not available. Figures in the parenthesis indicate the number of observation.

Table 2. Body measurement (cm or as stated) of BBG according to sex, generation and age group.

\begin{tabular}{|c|c|c|c|c|c|c|c|}
\hline \multirow[t]{2}{*}{ Parameters } & \multirow{2}{*}{$\begin{array}{l}\text { Age } \\
(\mathbf{m})\end{array}$} & \multirow[t]{2}{*}{ Sex } & \multicolumn{4}{|c|}{ Generations } & \multirow[t]{2}{*}{ Overall mean } \\
\hline & & & $\mathbf{G}_{\mathbf{0}}$ & $\mathbf{G}_{1}$ & $\mathbf{G}_{2}$ & $\mathbf{G}_{3}$ & \\
\hline \multirow[t]{6}{*}{ Body length } & $6-12$ & $\sigma^{1}$ & $\begin{array}{l}43.2 \pm 1.74 \\
(23)\end{array}$ & $47.9 \pm 0.98(21)$ & $43.8 \pm 0.81(27)$ & $45.0 \pm 2.00(30)$ & $44.9 \pm 0.72$ \\
\hline & & $q$ & $41.7 \pm 0.82(19)$ & $41.9 \pm 0.80(22)$ & $40.8 \pm 1.07(25)$ & $45.5 \pm 3.50(27)$ & $42.5 \pm 0.78$ \\
\hline & $12-24$ & $\sigma^{\lambda}$ & - & $54.2 \pm 1.67(15)$ & $48.3 \pm 1.80$ (19) & $47.0 \pm 1.58(20)$ & $49.8 \pm 1.28$ \\
\hline & & q & $\begin{array}{l}48.0 \pm 0.67 \\
(14))\end{array}$ & $52.3 \pm 1.02(17)$ & $47.1 \pm 1.05(21)$ & - & $49.1 \pm 0.62$ \\
\hline & $>24$ & 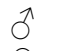 & - & $61.3 \pm 1.56(13)$ & - & - & $61.3 \pm 1.56$ \\
\hline & & q & $54.8 \pm 0.68(9)$ & $56.4 \pm 0.75(11)$ & $51.0 \pm 0.00(10)$ & - & $54.1 \pm 0.69$ \\
\hline \multirow[t]{6}{*}{ Heart girth } & $6-12$ & $\hat{\sigma}$ & $54.1 \pm 1.75(12)$ & $57.0 \pm 1.13(14)$ & $54.1 \pm 0.84(16)$ & $60.5 \pm 4.50(9)$ & $56.4 \pm 0.65$ \\
\hline & & q & $52.2 \pm 0.6(13)$ & $52.3 \pm 1.16(11)$ & $49.6 \pm 1.63(8)$ & $52.5 \pm 2.50(11)$ & $51.7 \pm 0.48$ \\
\hline & $12-24$ & $0^{\pi}$ & - & $66.6 \pm 1.52(9)$ & $60.8 \pm 1.43(6)$ & $57.3 \pm 3.64(11)$ & $61.6 \pm 1.02$ \\
\hline & & q & $57.7 \pm 0.50(10)$ & $64.2 \pm 1.27(8)$ & $59.7 \pm 2.17(9)$ & - & $60.5 \pm 0.48$ \\
\hline & $>24$ & $\hat{\sigma}$ & - & $72.3 \pm 1.35$ (13) & - & - & $72.3 \pm 1.35$ \\
\hline & & q & $69.6 \pm 0.87(12)$ & $69.8 \pm 1.16(17)$ & $65.0 \pm 0.00(9)$ & - & $68.1 \pm 0.82$ \\
\hline \multirow[t]{6}{*}{ Body weight } & $6-12$ & $\hat{0}$ & $10.9 \pm 0.94(19)$ & $14.6 \pm 0.79(12)$ & $12.1 \pm 0.55(16)$ & $15.4 \pm 2.95(13)$ & $13.3 \pm 0.52$ \\
\hline & & $q$ & $11.4 \pm 0.38(11)$ & $10.8 \pm 0.61(14)$ & $9.5 \pm 0.78(22)$ & $11.5 \pm 0.21(12)$ & $10.8 \pm 0.19$ \\
\hline & $12-24$ & $\hat{\sigma}$ & - & $22.7 \pm 1.64(21)$ & $16.3 \pm 1.49(11)$ & $14.6 \pm 2.26(9)$ & $17.9 \pm 1.43$ \\
\hline & & 우 & $14.9 \pm 0.39(11)$ & $20.4 \pm 1.13(9)$ & $\begin{array}{l}17.5 \pm 1.07 \\
(12)\end{array}$ & - & $17.6 \pm 0.29$ \\
\hline & $>24$ & $\sigma^{\pi}$ & - & $29.9 \pm 1.76(7)$ & - & - & $29.9 \pm 1.76$ \\
\hline & & q & $25.1 \pm 0.87(14)$ & $25.9 \pm 1.08(8)$ & $19.9 \pm 0.00(6)$ & - & $23.6 \pm 0.81$ \\
\hline \multirow[t]{6}{*}{ Wither height } & $6-12$ & $\hat{\sigma}$ & $46.7 \pm 1.22(11)$ & $50.2 \pm 0.93(10)$ & $47.8 \pm 0.87(15)$ & $48.3 \pm 2.75(9)$ & $48.3 \pm 0.65$ \\
\hline & & $q$ & $46.4 \pm 0.47$ (14) & $43.1 \pm 0.77$ (11) & $42.6 \pm 1.06(13)$ & $43.3 \pm 2.48(8)$ & $43.9 \pm 0.42$ \\
\hline & $12-24$ & $\hat{0}$ & - & $56.5 \pm 1.24(13)$ & $53.2 \pm 1.54(12)$ & $50.3 \pm 1.31(10)$ & $53.3 \pm 0.96$ \\
\hline & & q & $48.3 \pm 0.54(12)$ & $51.9 \pm 0.82(9)$ & $49.6 \pm 0.85(11)$ & - & $49.9 \pm 0.43$ \\
\hline & $>24$ & $\hat{0}$ & - & $59.7 \pm 1.26(12)$ & - & - & $59.7 \pm 1.26$ \\
\hline & & q & $52.9 \pm 0.42(11)$ & $53.6 \pm 0.61(16)$ & $50.0 \pm 0.00(10)$ & - & $52.2 \pm 0.39$ \\
\hline
\end{tabular}

$\mathrm{G}_{0}, \mathrm{G}_{1}, \mathrm{G}_{2}$ and $\mathrm{G}_{3}$ means foundation stock, $1^{\text {st }}$ generation, $2^{\text {nd }}$ generation and $3^{\text {rd }}$ generation, respectively; - means samples not available. Figures in the parenthesis indicate the number of observation. 
Asian J. Med. Biol. Res. 2018, 4 (1)

Table 3. Head measurement $(\mathrm{cm})$ of Black Bengal goat according to sex, generation and age group.

\begin{tabular}{|c|c|c|c|c|c|c|c|}
\hline \multirow[t]{2}{*}{ Parameters } & \multirow{2}{*}{$\begin{array}{l}\text { Age } \\
\text { (m) }\end{array}$} & \multirow[t]{2}{*}{ Sex } & \multicolumn{3}{|c|}{ Generations } & & \multirow[t]{2}{*}{ Overall mean } \\
\hline & & & $\mathbf{G}_{\mathbf{0}}$ & $\mathbf{G}_{1}$ & $\mathbf{G}_{2}$ & $\mathbf{G}_{3}$ & \\
\hline \multirow[t]{6}{*}{ Head length } & $6-12$ & $\hat{\sigma}$ & $14.5 \pm 0.42(12)$ & $15.3 \pm 0.35$ & $14.5 \pm 0.22(21)$ & $14.8 \pm 0.25(24)$ & $14.8 \pm 0.19$ \\
\hline & & $q$ & $14.0 \pm 0.23(10)$ & $14.0 \pm 0.40(11)$ & $13.4 \pm 0.52(12)$ & $13.3 \pm 0.75(18)$ & $13.7 \pm 0.18$ \\
\hline & $12-24$ & $\hat{\sigma}$ & - & $16.3 \pm 0.39(13)$ & $15.8 \pm 0.31(16)$ & $16.1 \pm 0.75$ & $16.1 \pm 0.26$ \\
\hline & & $q$ & $15.5 \pm 0.26(10)$ & $15.9 \pm 0.29(14)$ & $15.2 \pm 0.33$ & - & $15.5 \pm 0.22$ \\
\hline & $>24$ & $\hat{0}$ & - & $17.1 \pm 0.31(11)$ & - & - & $17.1 \pm 0.31$ \\
\hline & & q & $16.9 \pm 0.26(13)$ & $16.6 \pm 0.34(17)$ & $15.0 \pm 0.00(19)$ & - & $16.2 \pm 0.18$ \\
\hline \multirow[t]{6}{*}{ Head breadth } & $6-12$ & $\hat{O}$ & $10.1 \pm 0.33$ & $9.5 \pm 0.19(9)$ & $9.1 \pm 0.19(15)$ & $9.3 \pm 0.25(10)$ & $9.5 \pm 0.13$ \\
\hline & & $q$ & $9.0 \pm 0.19(12)$ & $8.6 \pm 0.14(13)$ & $8.4 \pm 0.20(16)$ & $8.8 \pm 0.25$ (17) & $8.7 \pm 0.12$ \\
\hline & $12-24$ & $\hat{\sigma}$ & - & $10.8 \pm 0.40(12)$ & $10.3 \pm 0.42(10)$ & $9.9 \pm 0.52(15)$ & $10.3 \pm 0.34$ \\
\hline & & q & $9.8 \pm 0.14(11)$ & $10.7 \pm 0.26(13)$ & $10.3 \pm 0.35$ & - & $10.3 \pm 0.12$ \\
\hline & $>24$ & $\hat{0}$ & - & $12.2 \pm 0.33(14)$ & - & - & $12.2 \pm 0.33$ \\
\hline & & $q$ & $11.2 \pm 0.14$ & $11.4 \pm 0.19$ & $12.0 \pm 0.00(9)$ & - & $11.5 \pm 0.11$ \\
\hline
\end{tabular}

$\mathrm{G}_{0}, \mathrm{G}_{1}, \mathrm{G}_{2}$ and $\mathrm{G}_{3}$ means foundation stock, $1^{\text {st }}$ generation, $2^{\text {nd }}$ generation and $3^{\text {rd }}$ generation, respectively; - means samples not available. Figures in the parenthesis indicate the number of observation.

Table 4. Horn measurement $(\mathrm{cm})$ of Black Bengal goat according to sex, generation and age group.

\begin{tabular}{|c|c|c|c|c|c|c|c|}
\hline \multirow[t]{2}{*}{ Parameters } & \multirow{2}{*}{$\begin{array}{l}\text { Age } \\
(\mathbf{m})\end{array}$} & \multirow[t]{2}{*}{ Sex } & \multicolumn{4}{|c|}{ Generations } & \multirow{2}{*}{$\begin{array}{l}\text { Overall } \\
\text { mean }\end{array}$} \\
\hline & & & $\mathbf{G}_{\mathbf{0}}$ & $\mathbf{G}_{1}$ & $\mathbf{G}_{2}$ & $\mathbf{G}_{3}$ & \\
\hline \multirow{6}{*}{$\begin{array}{l}\text { Left horn } \\
\text { length }\end{array}$} & $6-12$ & $\hat{0}$ & $3.8 \pm 0.32(12)$ & $5.9 \pm 0.43(14)$ & $4.5 \pm 0.27(15)$ & $4.0 \pm 0.00(19)$ & $4.6 \pm 0.23$ \\
\hline & & $q$ & $3.6 \pm 0.19(11)$ & $3.6 \pm 0.34(12)$ & $2.8 \pm 0.25(14)$ & - & $3.3 \pm 0.13$ \\
\hline & $12-24$ & 0 & - & $8.9 \pm 0.85(11)$ & $7.4 \pm 0.99(10)$ & $4.9 \pm 1.26(13)$ & $7.1 \pm 0.79$ \\
\hline & & q & $4.9 \pm 0.34(9)$ & $6.9 \pm 0.36(10)$ & $6.0 \pm 0.44(13)$ & - & $5.9 \pm 0.31$ \\
\hline & $>24$ & $\hat{0}$ & - & $12.4 \pm 1.74(12)$ & - & - & $12.4 \pm 1.74$ \\
\hline & & q & $7.5 \pm 0.38$ & $7.8 \pm 0.40(13)$ & $9.0 \pm 0.00(16)$ & - & $8.1 \pm 0.36$ \\
\hline \multirow{6}{*}{$\begin{array}{l}\text { Right horn } \\
\text { length }\end{array}$} & $6-12$ & $\hat{\sigma}$ & $4.0 \pm 0.29(12)$ & $6.0 \pm 0.40(14)$ & $4.6 \pm 0.29(15)$ & $5.2 \pm 0.85$ (19) & $5.0 \pm 0.26$ \\
\hline & & ㅇ & $3.6 \pm 0.25(11)$ & $3.9 \pm 0.40(12)$ & $2.5 \pm 0.50(14)$ & - & $3.3 \pm 0.22$ \\
\hline & $12-24$ & 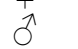 & - & $9.4 \pm 0.86(11)$ & $7.1 \pm 0.92(10)$ & $5.4 \pm 1.60(13)$ & $7.3 \pm 0.76$ \\
\hline & & q & $5.1 \pm 0.35(9)$ & $6.9 \pm 0.38(10)$ & $6.1 \pm 0.49(13)$ & - & $6.0 \pm 0.33$ \\
\hline & $>24$ & $\hat{0}$ & - & $13.2 \pm 1.78(12)$ & - & - & $13.2 \pm 1.78$ \\
\hline & & q & $7.3 \pm 0.32(11)$ & $7.5 \pm 0.34$ (13) & $9.0 \pm 0.00(16)$ & - & $7.9 \pm 0.29$ \\
\hline \multirow{6}{*}{$\begin{array}{l}\text { Left horn } \\
\text { circumference }\end{array}$} & $6-12$ & $\hat{0}$ & $4.9 \pm 0.31(12)$ & $6.2 \pm 0.26$ & $5.3 \pm 0.16(15)$ & $5.5 \pm 0.00(19)$ & $5.5 \pm 0.13$ \\
\hline & & q & $4.5 \pm 0.20(11)$ & $4.4 \pm 0.22(12)$ & $3.9 \pm 0.10(14)$ & - & $4.3 \pm 0.08$ \\
\hline & $12-24$ & $\hat{o}$ & - & $7.3 \pm 0.45$ & $6.5 \pm 0.58(10)$ & $5.0 \pm 0.35(13)$ & $6.3 \pm 0.33$ \\
\hline & & 운 & $5.1 \pm 0.13(9)$ & $5.6 \pm 0.18(10)$ & $5.2 \pm 0.19(13)$ & - & $5.3 \pm 0.12$ \\
\hline & $>24$ & $\hat{\sigma}$ & - & $9.0 \pm 0.57(12)$ & - & - & $9.0 \pm 0.57$ \\
\hline & & o & $6.1 \pm 0.17(11)$ & $6.3 \pm 0.17(13)$ & $6.5 \pm 0.00(16)$ & - & $6.3 \pm 0.15$ \\
\hline \multirow{6}{*}{$\begin{array}{l}\text { Right horn } \\
\text { circumference }\end{array}$} & $6-12$ & $\hat{o}$ & $4.9 \pm 0.31(11)$ & $6.3 \pm 0.26$ & $5.6 \pm 0.19(15)$ & $6.5 \pm 1.00(19)$ & $5.8 \pm 0.22$ \\
\hline & & q & $4.5 \pm 0.22(11)$ & $4.2 \pm 0.17$ (12) & $3.5 \pm 0.00(14)$ & - & $4.1 \pm 0.14$ \\
\hline & $12-24$ & $\hat{0}$ & - & $7.5 \pm 0.48$ & $6.8 \pm 0.47(10)$ & $5.4 \pm 0.31(13)$ & $6.6 \pm 0.29$ \\
\hline & & q & $4.9 \pm 0.15(9)$ & $5.8 \pm 0.15(10)$ & $5.2 \pm 0.23(13)$ & - & $5.3 \pm 0.13$ \\
\hline & $>24$ & $\hat{0}$ & - & $9.7 \pm 0.48(12)$ & - & - & $9.7 \pm 0.48$ \\
\hline & & ㅇ & $6.0 \pm 0.00(11)$ & $6.1 \pm 0.17$ & $6.0 \pm 0.00(16)$ & - & $6.0 \pm 0.17$ \\
\hline \multirow[t]{6}{*}{ Horn distance } & $6-12$ & $\hat{0}$ & $3.6 \pm 0.33$ (11) & $3.2 \pm 0.16(14)$ & $3.5 \pm 0.13(15)$ & $4.0 \pm 0.50(19)$ & $3.6 \pm 0.14$ \\
\hline & & q & $3.4 \pm 0.16(11)$ & $3.7 \pm 0.10(12)$ & $3.9 \pm 0.16(14)$ & $3.3 \pm 0.25(16)$ & $3.6 \pm 0.13$ \\
\hline & $12-24$ & $\hat{0}$ & - & $2.8 \pm 0.14(11)$ & $3.2 \pm 0.29(10)$ & $4.0 \pm 0.29(13)$ & $3.3 \pm 0.13$ \\
\hline & & q & $3.3 \pm 0.12(9)$ & $3.2 \pm 0.07$ (10) & $3.2 \pm 0.11(13)$ & - & $3.2 \pm 0.06$ \\
\hline & $>24$ & $\hat{0}$ & - & $2.8 \pm 0.24(12)$ & - & - & $2.8 \pm 0.24$ \\
\hline & & q & $3.2 \pm 0.07(11)$ & $2.9 \pm 0.09(13)$ & $3.0 \pm 0.00(16)$ & - & $3.0 \pm 0.06$ \\
\hline
\end{tabular}

$\mathrm{G}_{0}, \mathrm{G}_{1}, \mathrm{G}_{2}$ and $\mathrm{G}_{3}$ means foundation stock, $1^{\text {st }}$ generation, $2^{\text {nd }}$ generation and $3^{\text {rd }}$ generation, respectively; - means samples not available. 
Table 5. Ear measurement (cm) of Black Bengal goat according to sex, generation and age group.

\begin{tabular}{|c|c|c|c|c|c|c|c|}
\hline \multirow[t]{2}{*}{ Parameters } & \multirow{2}{*}{$\begin{array}{l}\text { Age } \\
\text { (m) }\end{array}$} & \multirow[t]{2}{*}{ Sex } & \multicolumn{4}{|c|}{ Generations } & \multirow[t]{2}{*}{ Overall mean } \\
\hline & & & $\mathbf{G}_{\mathbf{0}}$ & $\mathbf{G}_{1}$ & $\mathbf{G}_{2}$ & $\mathbf{G}_{3}$ & \\
\hline \multirow[t]{6}{*}{ Left ear length } & $6-12$ & $\hat{0}$ & $9.7 \pm 0.99(11)$ & $10.1 \pm 0.24(14)$ & $11.0 \pm 0.18(15)$ & $11.3 \pm 0.25(19)$ & $10.5 \pm 0.17$ \\
\hline & & q & $11.3 \pm 0.32(11)$ & $10.7 \pm 0.20$ & $10.8 \pm 0.19$ (14) & $11.3 \pm 0.75(16)$ & $11.0 \pm 0.15$ \\
\hline & $12-24$ & $\hat{\sigma}$ & - & $11.3 \pm 0.22$ & $11.6 \pm 0.23(10)$ & $11.3 \pm 0.52(13)$ & $11.4 \pm 0.18$ \\
\hline & & q & $11.7 \pm 0.19$ (19) & $11.6 \pm 0.17(10)$ & $11.1 \pm 0.29(13)$ & - & $11.5 \pm 0.16$ \\
\hline & $>24$ & $\hat{\sigma}$ & - & $10.9 \pm 0.28(12)$ & - & - & $10.9 \pm 0.28$ \\
\hline & & q & $12.0 \pm 0.13(11)$ & $12.0 \pm 0.14(13)$ & $11.0 \pm 0.00(16)$ & - & $11.7 \pm 0.12$ \\
\hline \multirow{6}{*}{$\begin{array}{l}\text { Right ear } \\
\text { length }\end{array}$} & $6-12$ & $\hat{\sigma}$ & $9.6 \pm 0.68(11)$ & $10.9 \pm 0.22(14)$ & $11.0 \pm 0.18(15)$ & $11.5 \pm 0.50(19)$ & $10.8 \pm 0.14$ \\
\hline & & q & $11.1 \pm 0.24(11)$ & $10.8 \pm 0.24(12)$ & $11.0 \pm 0.23(14)$ & $10.8 \pm 0.75(16)$ & $11.0 \pm 0.19$ \\
\hline & $12-24$ & $\hat{\sigma}$ & - & $11.6 \pm 0.17(11)$ & $11.7 \pm 0.21(10)$ & $11.0 \pm 0.41(13)$ & $11.4 \pm 0.16$ \\
\hline & & q & $11.8 \pm 0.19$ (19) & $11.6 \pm 0.19(10)$ & $11.3 \pm 0.21(13)$ & - & $11.6 \pm 0.17$ \\
\hline & $>24$ & $\hat{o}$ & - & $11.1 \pm 0.34(12)$ & - & - & $11.1 \pm 0.34$ \\
\hline & & o & $12.0 \pm 0.15(11)$ & $12.0 \pm 0.13$ & $11.0 \pm 0.00(16)$ & - & $11.7 \pm 0.12$ \\
\hline \multirow{6}{*}{$\begin{array}{l}\text { Left ear } \\
\text { breadth }\end{array}$} & $6-12$ & $\hat{o}$ & $4.8 \pm 0.20(11)$ & $5.4 \pm 0.13$ & $5.5 \pm 0.08(15)$ & $5.3 \pm 0.25$ (19) & $5.3 \pm 0.11$ \\
\hline & & q & $5.3 \pm 0.09(11)$ & $5.2 \pm 0.11(12)$ & $5.2 \pm 0.15$ & $5.3 \pm 0.25(16)$ & $5.3 \pm 0.09$ \\
\hline & $12-24$ & $\hat{o}$ & - & $5.7 \pm 0.10$ & $5.8 \pm 0.12(10)$ & $5.5 \pm 0.29(13)$ & $5.7 \pm 0.08$ \\
\hline & & q & $5.5 \pm 0.09$ (19) & $5.7 \pm 0.11(10)$ & $5.5 \pm 0.14(13)$ & - & $5.6 \pm 0.06$ \\
\hline & $>24$ & $\hat{0}$ & - & $5.9 \pm 0.18(12)$ & - & - & $5.9 \pm 0.18$ \\
\hline & & q & $5.9 \pm 0.05(11)$ & $5.9 \pm 0.08$ (13) & $5.5 \pm 0.00(16)$ & - & $5.8 \pm 0.04$ \\
\hline \multirow{6}{*}{$\begin{array}{l}\text { Right ear } \\
\text { breadth }\end{array}$} & $6-12$ & $\hat{0}$ & $5.0 \pm 0.32(11)$ & $5.3 \pm 0.11(14)$ & $5.4 \pm 0.08(15)$ & $5.3 \pm 0.25$ (19) & $5.3 \pm 0.06$ \\
\hline & & q & $5.3 \pm 0.09(11)$ & $5.2 \pm 0.10(12)$ & $5.2 \pm 0.17(14)$ & $5.3 \pm 0.25$ & $5.3 \pm 0.07$ \\
\hline & $12-24$ & $\hat{0}$ & - & $5.7 \pm 0.09(11)$ & $5.7 \pm 0.17$ & $5.5 \pm 0.29(13)$ & $5.6 \pm 0.08$ \\
\hline & & 우 & $5.5 \pm 0.10(19)$ & $5.7 \pm 0.10(10)$ & $5.6 \pm 0.13(13)$ & - & $5.6 \pm 0.09$ \\
\hline & $>24$ & $\hat{\sigma}$ & - & $5.9 \pm 0.20(12)$ & - & - & $5.9 \pm 0.20$ \\
\hline & & 우 & $5.9 \pm 0.05(11)$ & $5.8 \pm 0.08(13)$ & $5.5 \pm 0.00(16)$ & - & $5.7 \pm 0.04$ \\
\hline
\end{tabular}

$\mathrm{G}_{0}, \mathrm{G}_{1}, \mathrm{G}_{2}$ and $\mathrm{G}_{3}$ means foundation stock, $1^{\text {st }}$ generation, $2^{\text {nd }}$ generation and $3^{\text {rd }}$ generation, respectively; - means samples not available.

\subsection{Horn distance}

Horn distance of different ages male and female goats are shown in Table 4. The average horn distance of adult goats ranged from 3 to $4 \mathrm{~cm}$. No available report was found in literature on horn circumference and horn distance in BBG.

\subsection{Ear length and breadth}

The left and right ear length and breadth of male and female goats at different age group in different generation are presented in Table 5. Ear length, ear breadth, neck length and neck breadth measurement of BBG are shown in Figure 3. The average ear length of adult ( $>6$ months) male and female goats were 10.5 and 11.0 respectively (range 9.6 to $12 \mathrm{~cm}$ ). Female goats had higher ear length than male goats (Table 5). This result is very close with the result of Chowdhury and Faruqu, $2001 \mathrm{~b}$ who reported that the ear length ranges from 11.6 to $13.3 \mathrm{~cm}$ in goats of $<7$ months old age. Acharya, 1982 and Devendra and Burns, 1983 also stated that the ear of BBG is short [13.8 cm (11.5 to14.1 cm)], flat, carried horizontally. They reported that average ear length images from 11.5 to 14.1 with an average of 13.8

\subsection{Neck length and breadth}

Neck length and breadth of male and female goats at different age group and generation are shown in Table 6 . Average neck length of adult (>24 months) female goats was higher $(16.6 \pm 0.34 \mathrm{~cm})$ than male goats $(16.3 \pm 0.50$ $\mathrm{cm})$. But neck breadth was higher in male goats $(42.4 \pm 1.70 \mathrm{~cm})$ than in female goats $(29.2 \pm 0.54 \mathrm{~cm})$. No such report was cited on neck measurement of Black Bengal goats.

\subsection{Tail length and breadth}

Tail length and breadth of Black Bengal goats are presented in Table 6. Tail length, tail breadth, leg length, testis length and testis breadth measurement of BBG are shown in Figure 4. Tail length of Black Bengal goats ranged from $8.0 \pm 0.52$ to $11.1 \pm 0.43 \mathrm{~cm}$ and tail breadth ranged from $2.1 \pm 0.10$ to $3.6 \pm 0.43 \mathrm{~cm}$ for different age and sex group. Literatures on these measurements are not available to compare with this study. 


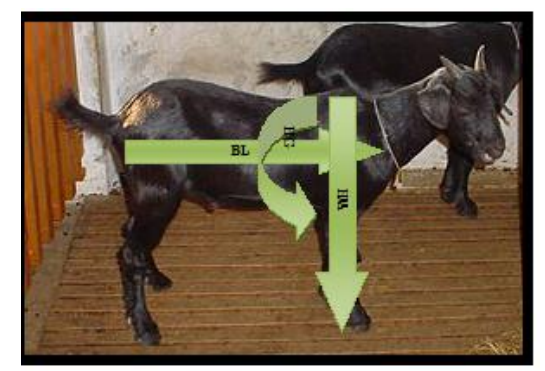

Figure 1. Different body measurement of BBG: BL=Body Length, HG=Heart Girth and WH= Wither Height
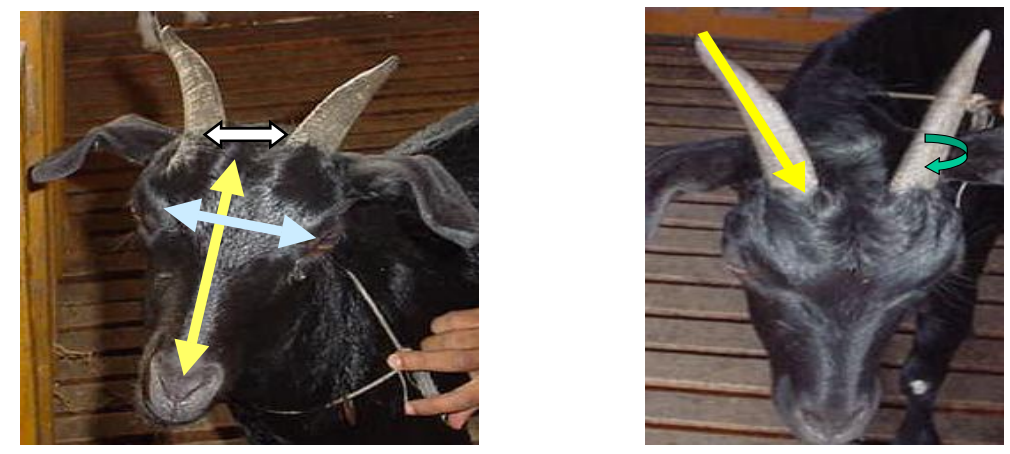

Figure 2. Head length, breadth, Horn length, circumference and Horn distance measurement of BBG.
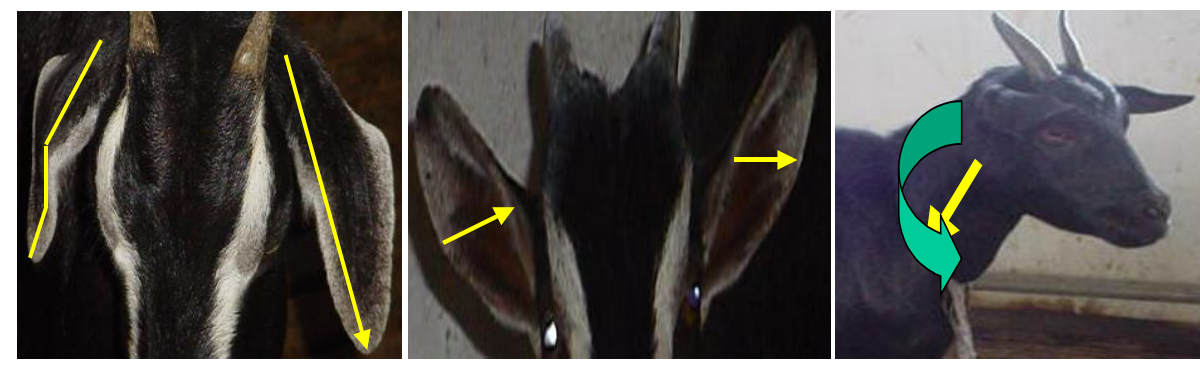

Figure 3. Ear length, ear breadth, neck length and neck breadth measurement of BBG.
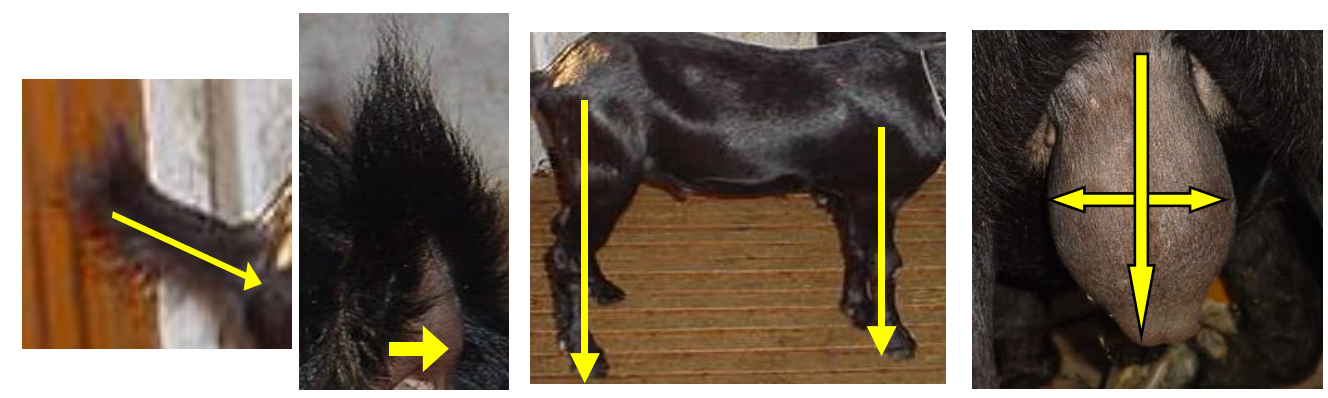

Figure 4. Tail length, tail breadth, leg length, testis length and testis breadth measurement of BBG.

\subsection{Leg length}

Fore and hind leg length of male and female goats at different age group in different generations are presented in Table 6. Average male foreleg length in $>24$ months of age possessed higher $(38.2 \pm 0.81 \mathrm{~cm})$ than that of female $(37.1 \pm 0.53 \mathrm{~cm})$. On the other hand, average hind leg length in the same age was higher in male $(48.6 \pm 0.87 \mathrm{~cm})$ than female goats $(43.4 \pm 0.35 \mathrm{~cm})$. These results are in agreement with the results of Chowdhury and Faruque, 2001b who reported that fore and hind leg length is higher in males (38.3 to $40.9 \mathrm{~cm}$ and 44.1 to 
$46.2 \mathrm{~cm}$, respectively) than in females ( 37.5 to $42.1 \mathrm{~cm}$ and 38.9 to $43.5 \mathrm{~cm}$, respectively). Kibria and Kamal, 1990 also reported that the leg length of BBG ranges from 39.5 to $43.0 \mathrm{~cm}$.

Table 6. Neck, tail and leg measurement $(\mathrm{cm})$ of Black Bengal goat according to sex, generation and age group.

\begin{tabular}{|c|c|c|c|c|c|c|c|}
\hline \multirow[t]{2}{*}{ Parameters } & \multirow{2}{*}{$\begin{array}{l}\text { Age } \\
\text { (m) }\end{array}$} & \multirow[t]{2}{*}{ Sex } & \multicolumn{4}{|c|}{ Generations } & \multirow[t]{2}{*}{ Overall mean } \\
\hline & & & $\mathbf{G}_{\mathbf{0}}$ & $\mathbf{G}_{\mathbf{1}}$ & $\mathbf{G}_{\mathbf{2}}$ & $\mathbf{G}_{3}$ & \\
\hline \multirow[t]{6}{*}{ Neck length } & $6-12$ & 0 & $13.4 \pm 0.50(11)$ & $14.8 \pm 0.30(14)$ & $13.4 \pm 0.27(15)$ & $15.0 \pm 2.00(19)$ & $14.2 \pm 0.24$ \\
\hline & & 우 & $13.5 \pm 0.44(11)$ & $13.9 \pm 0.40(12)$ & $13.3 \pm 0.45$ (14) & $14.3 \pm 0.25(16)$ & $13.8 \pm 0.23$ \\
\hline & $12-24$ & $0^{2}$ & - & $15.2 \pm 0.30$ & - & - & $15.2 \pm 0.30$ \\
\hline & & 우 & $14.8 \pm 0.30(12)$ & $16.5 \pm 0.34(17)$ & - & - & $15.7 \pm 0.28$ \\
\hline & $>24$ & o & - & $16.3 \pm 0.50(18)$ & - & - & $16.3 \pm 0.50$ \\
\hline & & 우 & $16.2 \pm 0.35(14)$ & $16.9 \pm 0.37(20)$ & - & - & $16.6 \pm 0.34$ \\
\hline \multirow[t]{6}{*}{ Neck breadth } & $6-12$ & $\hat{0}$ & $24.2 \pm 0.73(11)$ & $30.8 \pm 1.18(12)$ & $27.6 \pm 0.85(15)$ & $31.3 \pm 1.75$ (19) & $28.5 \pm 0.68$ \\
\hline & & 우 & $22.9 \pm 0.34(11)$ & $22.3 \pm 0.52(12)$ & $21.5 \pm 0.54(14)$ & $23.5 \pm 0.50(16)$ & $22.6 \pm 0.32$ \\
\hline & $12-24$ & $\hat{0}$ & - & $31.3 \pm 1.18(15)$ & - & - & $31.3 \pm 1.18$ \\
\hline & & q & $24.3 \pm 0.39(12)$ & $27.5 \pm 0.61(17)$ & - & - & $25.9 \pm 0.37$ \\
\hline & $>24$ & $\hat{0}$ & - & $42.4 \pm 1.70(18)$ & - & - & $42.4 \pm 1.70$ \\
\hline & & of & $28.8 \pm 0.55(14)$ & $29.6 \pm 0.95(20)$ & - & - & $29.2 \pm 0.54$ \\
\hline \multirow[t]{6}{*}{ Tail length } & $6-12$ & $0^{\lambda}$ & $8.0 \pm 0.52(11)$ & $9.5 \pm 0.19(12)$ & $9.1 \pm 0.25(15)$ & $9.5 \pm 0.50(19)$ & $9.0 \pm 0.16$ \\
\hline & & 우 & $8.4 \pm 0.24(11)$ & $8.8 \pm 0.19(12)$ & $8.4 \pm 0.40(14)$ & $9.0 \pm 1.00(16)$ & $8.7 \pm 0.18$ \\
\hline & $12-24$ & 0 & - & $9.4 \pm 0.30$ (14) & $10.2 \pm 0.35$ & $11.1 \pm 0.43(19)$ & $10.2 \pm 0.28$ \\
\hline & & o & $8.9 \pm 0.19(12)$ & $9.7 \pm 0.30(17)$ & $9.5 \pm 0.30(18)$ & - & $9.4 \pm 0.18$ \\
\hline & $>24$ & $\hat{0}$ & - & $9.2 \pm 0.30(18)$ & - & - & $9.2 \pm 0.30$ \\
\hline & & 우 & $9.8 \pm 0.19(14)$ & $9.5 \pm 0.23(20)$ & $8.0 \pm 0.00(22)$ & - & $9.1 \pm 0.16$ \\
\hline \multirow[t]{6}{*}{ Tail breadth } & $6-12$ & $0^{\pi}$ & $2.1 \pm 0.10(11)$ & $2.7 \pm 0.08(12)$ & $2.7 \pm 0.08(15)$ & $2.8 \pm 0.10(19)$ & $2.6 \pm 0.06$ \\
\hline & & q & $2.9 \pm 0.06(11)$ & $3.1 \pm 0.16(12)$ & $3.1 \pm 0.18(14)$ & $3.3 \pm 0.25(16)$ & $3.1 \pm 0.04$ \\
\hline & $12-24$ & $0^{2}$ & - & $3.0 \pm 0.12(14)$ & $2.8 \pm 0.14$ (17) & $3.6 \pm 0.43(19)$ & $3.1 \pm 0.11$ \\
\hline & & 우 & $3.0 \pm 0.07$ (12) & $3.5 \pm 0.11(17)$ & $3.0 \pm 0.11(18)$ & - & $3.2 \pm 0.05$ \\
\hline & $>24$ & $0^{\lambda}$ & - & $2.9 \pm 0.09$ (18) & - & - & $2.9 \pm 0.09$ \\
\hline & & 우 & $3.7 \pm 0.09$ (14) & $3.5 \pm 0.11(20)$ & $3.0 \pm 0.00(22)$ & - & $3.4 \pm 0.07$ \\
\hline \multirow{6}{*}{$\begin{array}{l}\text { Foreleg } \\
\text { length }\end{array}$} & $6-12$ & o & $31.6 \pm 1.60(11)$ & $34.3 \pm 0.64(12)$ & $32.6 \pm 0.53(15)$ & $35.3 \pm 0.85$ (19) & $33.5 \pm 0.50$ \\
\hline & & 우 & $33.1 \pm 0.74(11)$ & $32.1 \pm 1.38(12)$ & $30.3 \pm 0.75(14)$ & - & $31.8 \pm 0.32$ \\
\hline & $12-24$ & $0^{1}$ & - & $38.2 \pm 0.82(17)$ & $34.9 \pm 0.77(18)$ & - & $36.6 \pm 0.72$ \\
\hline & & 오 & $33.1 \pm 0.58(17)$ & $38.0 \pm 0.64(15)$ & $34.9 \pm 0.87(18)$ & $35.3 \pm 0.85(20)$ & $35.3 \pm 0.57$ \\
\hline & $>24$ & $\hat{O}^{\hat{2}}$ & - & $38.2 \pm 0.81(18)$ & - & - & $38.2 \pm 0.81$ \\
\hline & & 오 & $37.4 \pm 0.55(15)$ & $37.9 \pm 0.71(16)$ & $36.0 \pm 0.00$ (19) & - & $37.1 \pm 0.53$ \\
\hline \multirow{6}{*}{$\begin{array}{l}\text { Hind leg } \\
\text { length }\end{array}$} & $6-12$ & o & $38.0 \pm 0.89(11)$ & $42.5 \pm 0.75(12)$ & $39.8 \pm 0.65(15)$ & $38.5 \pm 2.50(19)$ & $39.7 \pm 0.59$ \\
\hline & & 오 & $39.5 \pm 0.61(11)$ & $37.7 \pm 1.11(12)$ & $35.8 \pm 0.99(14)$ & $35.0 \pm 2.00(16)$ & $37.0 \pm 0.58$ \\
\hline & $12-24$ & o & - & $47.6 \pm 0.85$ (17) & $45.4 \pm 1.31(18)$ & $43.0 \pm 1.08(20)$ & $45.3 \pm 0.85$ \\
\hline & & 우 & $40.4 \pm 0.63(15)$ & $43.8 \pm 0.58(16)$ & $42.2 \pm 0.74(19)$ & - & $42.1 \pm 0.56$ \\
\hline & $>24$ & $0^{2}$ & - & $48.6 \pm 0.87(18)$ & - & - & $48.6 \pm 0.87$ \\
\hline & & 우 & $43.8 \pm 0.36(15)$ & $44.5 \pm 0.66(16)$ & $42.0 \pm 0.00(19)$ & - & $43.4 \pm 0.35$ \\
\hline
\end{tabular}

$\mathrm{G}_{0}, \mathrm{G}_{1}, \mathrm{G}_{2}$ and $\mathrm{G}_{3}$ means foundation stock, $1^{\text {st }}$ generation, $2^{\text {nd }}$ generation and $3^{\text {rd }}$ generation, respectively; - means samples not available. Figures in the parenthesis indicate the number of observation.

\subsection{Udder length and breadth}

Udder length and breadth of different age groups of female goats are presented in Table.7. From the Table 7, it appears that average udder length and breadth of BBGs were $7.5 \pm 0.24,11.7 \pm 0.44$ and $14.3 \pm 0.46$ and $5.9 \pm 0.16$, $7.4 \pm 0.28$ and $7.8 \pm 0.23 \mathrm{~cm}$, respectively for age groups $6-12,12-24$ and $>24$ months of age. No available literature was cited on mammary gland of BBG. Udder length may be varied due to age and hand milking of doe.

\subsection{Teat length and circumference}

Teat length and circumference of Black Bengal goats in different age group and generation are shown in Table 7. The average teat length of adult goats $(6-12,12-24$ and $>24$ months of age groups) were $2.6 \pm 0.07,3.8 \pm 0.14$ and $4.9 \pm 0.15 \mathrm{~cm}$ and circumference were $3.6 \pm 0.17,5.4 \pm 0.19$ and $7.4 \pm 0.22 \mathrm{~cm}$ respectively for those age groups. Teat length and circumference depends upon age and milking of doe. Literatures are not available to compare for this trait with others. 
3.15. Testis length and breadth

Testis length and breadth in different age groups of male are presented in Table 7 . The average testis length and breadth in adult males were $9.1 \pm 0.18,7.5 \pm 0.48$ and $9.8 \pm 0.49$ and $6.8 \pm 0.13,5.2 \pm 0.27$ and $6.3 \pm 0.32, \mathrm{~cm}$ respectively for 6-12, 12-24 and >24 months of ages groups. There appears to have no more literatures on this trait.

Table 7. Udder, teat and testes measurement $(\mathrm{cm})$ of Black Bengal goat according to generation and age group.

\begin{tabular}{|c|c|c|c|c|c|c|c|}
\hline \multirow[t]{2}{*}{ Parameters } & \multirow{2}{*}{$\begin{array}{l}\text { Age } \\
(\mathbf{m})\end{array}$} & \multirow[t]{2}{*}{ Sex } & \multicolumn{4}{|c|}{ Generations } & \multirow[t]{2}{*}{ Overall mean } \\
\hline & & & $\mathbf{G}_{\mathbf{0}}$ & $\mathbf{G}_{1}$ & $\mathbf{G}_{2}$ & $\mathbf{G}_{3}$ & \\
\hline \multirow[t]{3}{*}{ Udder length } & $6-12$ & 우 & $9.2 \pm 0.58(12)$ & $8.0 \pm 0.47(13)$ & $6.4 \pm 0.39(15)$ & $6.3 \pm 0.25(17)$ & $7.5 \pm 0.24$ \\
\hline & $12-24$ & 우 & $12.2 \pm 0.46(13)$ & $12.1 \pm 0.54(16)$ & $10.7 \pm 0.63(19)$ & - & $11.7 \pm 0.44$ \\
\hline & $>24$ & q & $14.0 \pm 0.48$ (14) & $14.9 \pm 0.58(14)$ & $14.0 \pm 0.00(16)$ & - & $14.3 \pm 0.46$ \\
\hline \multirow[t]{3}{*}{ Udder breadth } & $6-12$ & o & $6.7 \pm 0.34(12)$ & $6.3 \pm 0.39(13)$ & $5.4 \pm 0.18(15)$ & $5.5 \pm 0.00(17)$ & $5.9 \pm 0.16$ \\
\hline & $12-24$ & q & $8.1 \pm 0.29(13)$ & $7.4 \pm 0.36(16)$ & $6.8 \pm 0.32(19)$ & - & $7.4 \pm 0.28$ \\
\hline & $>24$ & 우 & $7.9 \pm 0.25(14)$ & $8.5 \pm 0.37$ (14) & $7.0 \pm 0.00(16)$ & - & $7.8 \pm 0.23$ \\
\hline \multirow[t]{3}{*}{ Teat length } & $6-12$ & 우 & $2.9 \pm 0.15(12)$ & $2.8 \pm 0.24(13)$ & $2.2 \pm 0.08(15)$ & $2.3 \pm 0.25$ (17) & $2.6 \pm 0.07$ \\
\hline & $12-24$ & q & $3.4 \pm 0.15(13)$ & $4.5 \pm 0.40(16)$ & $3.4 \pm 0.23$ (19) & - & $3.8 \pm 0.14$ \\
\hline & $>24$ & q & $4.3 \pm 0.18(14)$ & $4.4 \pm 0.30$ & $6.0 \pm 0.00(16)$ & - & $4.9 \pm 0.15$ \\
\hline Teat & $6-12$ & q & $4.3 \pm 0.21$ (19) & $3.6 \pm 0.18(20)$ & $3.1 \pm 0.20(21)$ & $3.5 \pm 0.50(22)$ & $3.6 \pm 0.17$ \\
\hline \multirow[t]{2}{*}{ circumference } & $12-24$ & q & $5.0 \pm 0.21(17)$ & $6.0 \pm 0.40(18)$ & $5.1 \pm 0.27(19)$ & - & $5.4 \pm 0.19$ \\
\hline & $>24$ & q & $6.0 \pm 0.25(19)$ & $6.3 \pm 0.38$ (19) & $10.0 \pm 0.00(18)$ & - & $7.4 \pm 0.22$ \\
\hline \multirow[t]{3}{*}{ Testis length } & $6-12$ & $\hat{o}$ & $9.1 \pm 0.24(22)$ & $9.4 \pm 0.20(23)$ & $8.7 \pm 0.20(24)$ & $9.3 \pm 0.25(24)$ & $9.1 \pm 0.18$ \\
\hline & $12-24$ & $\widehat{0}$ & - & $11.0 \pm 0.74(21)$ & $9.0 \pm 0.67(23)$ & $2.5 \pm 0.50(24)$ & $7.5 \pm 0.48$ \\
\hline & $>24$ & $\hat{0}$ & - & $9.8 \pm 0.49(22)$ & - & - & $9.8 \pm 0.49$ \\
\hline \multirow[t]{3}{*}{ Testis breadth } & $6-12$ & $\hat{0}$ & $6.4 \pm 0.29(22)$ & $6.7 \pm 0.17(23)$ & $6.5 \pm 0.16(24)$ & $7.5 \pm 0.00(24)$ & $6.8 \pm 0.13$ \\
\hline & $12-24$ & $\hat{0}$ & - & $6.8 \pm 0.29(21)$ & $6.3 \pm 0.44(23)$ & $2.5 \pm 0.50(24)$ & $5.2 \pm 0.27$ \\
\hline & $>24$ & $\hat{0}$ & - & $6.3 \pm 0.32(22)$ & - & - & $6.3 \pm 0.32$ \\
\hline
\end{tabular}

$\mathrm{G}_{0}, \mathrm{G}_{1}, \mathrm{G}_{2}$ and $\mathrm{G}_{3}$ means foundation stock, $1^{\text {st }}$ generation, $2^{\text {nd }}$ generation and $3^{\text {rd }}$ generation, respectively; - means samples not available. Figures in the parenthesis indicate the number of observation.

\section{Conclusions}

The results on different morphometric measurements obtained in this study showing that most of the parameters in male are higher than those in females and it increases linearly with increasing ages.

\section{Conflict of interest}

None to declare.

\section{References}

Acharya RM, 1982. Goat breeds. In: Sheep and goat breeds of India. FAO Animal Production and Health Paper 30. Food and Agriculture Organization of the United Nations, Rome, Italy. pp. 51-58.

Bhattacharya B, TK Ghosh, R Duttahupta and DN Maitra, 1984. Estimation of body weight in Black Bengal goats from body measurements. Indian Vet. J., 61: 406-408.

Chowdhury SA and S Faruque, 2001a. Improvement of Black Bengal goat through Selective Breeding. Bangladesh Livestock Research Institute, Savar and Bangladesh Agricultural Research Council, Farmgate, Dhaka, Bangladesh. pp.16-17.

Chowdhury SA and S Faruque, 2001b. Report on Improvement of Black Bengal goat through selective breeding. Bangladesh Livestock Research Institute, Savar, Dhaka, Bangladesh. pp.1-62..

Chowdhury SA, 2002. Goat: Our Natural Resource and Development Opportunities. Proceedings of the workshop on Poverty Alleviation Through Goat Production: National Program. Bangladesh Livestock Research Institute, Savar, Dhaka, Bangladesh. pp. 1-51.

Devendra C and Burns M, 1983 .Goats Production in the Tropics ( Revised edn.). Tecl Comm., Bureaux of Animal Breeding and Genetics, Commonwealth Agricultural Bereaux, England ,pp. 183.

Husain SS, 1993. A Study on the productive performance and genetic potentials of Black Bengal goats. PhD thesis, Department of Animal Breeding and Genetics, Bangladesh Agricultural University, Mymensingh, Bangladesh. pp.1-178. 
Husain SS, MR Amin and ABMM Islam, 1998. Goat productions and its breeding strategy in Bangladesh. Proc. Ist National workshop on Animal Breeding held on 26 Nov. 1998, Bangladesh Agricultural University, Mymensigh, Bangladesh. pp 17-36.

Husain SS, P Horst and ABMM Islam, 1996. Phenotypic selection on the improvement of growth performance of Black Bengal Kids. Asian-Australas. J. Anim. Sci., 9: 149-153.

Islam M, TN Nahar and S Hoq, 1991. Prospect of goat production in Bangladesh. Asian Livestock Livest. 16: 85-95.

Kibria SS and MM Kamal, 1990. Performance of Black Bengal goat under stall fed condition. Animal Production Research Division. Bangladesh Livestock Research Institute, Savar, Dhaka. pp.1-42.

Mukherjee DK, CSP Singh and HR Mishra, 1979. A note on some phenotypic parameters in Grey and Brown Bengal goats. Indian J. Anim. Sci., 49: 671-673.

Prasad B, CSP Singh and HR Mishra, 1981. Note on body weight-measurement relationship in Black Bengal goats. Indian J. Anim. Sci., 51: 880-882.

Singh DK, CSP Singh and K Jha, 1991. Post weaning survivability in kids under village conditions. Indian J. Anim. Sci., 61: 228-229.

Singh DK, RA Singh, MP Sinha and CSP Singh, 1986. Post partum oestrus in Black Bengal goats. Indian J. Anim. Sci., 56: 232-234.

Singh NR, SC Mohanty and M Mishra, 1987. Prediction of body weight from body measurement in Black Bengal goats. Indian J. Anim. Prod. and Manag., 3: 46-49. 